\title{
CLOSE-TO-CONVEX FUNCTIONS AND LINEAR-INVARIANT FAMILIES
}

\author{
WOLFRAM KOEPF
}

1. Introduction. Landau showed in 1925 [6] that in the class $S$ of normalized schlicht functions on the unit disk we can get a distortion theorem for the $n$-th derivative if we have ensured the first $n$ Bieberbach coefficient estimates to be correct.

We shall modify this result for linear-invariant families. Families of closeto-convex functions and of functions of bounded boundary rotation will be showed to be linear-invariant.

Because of the coefficient estimate for close-to-convex functions and functions of bounded boundary rotation derived by Aharonov and Friedland [1], it is possible to get the distortion theorem for the $n$-th derivative for all $n$, but here we obtain the same conclusion more elementarily (and without using the linear-invariance), just because the coefficient estimate is given for all $n$.

All functions $f$ considered here are analytic functions on the unit disk with normalization $f(0)=0, f^{\prime}(0)=1$, and they are locally schlicht, i.e., $\left\{z \mid f^{\prime}(z)=0\right\}=\emptyset$. Let $N$ be the class of such functions.

Pommerenke defined a linear-invariant family in [9] and showed some properties of such families. A subset $F$ of $N$ is called linear-invariant if it is closed under the re-normalized composition with a schlicht automorphism of the unit disk. If the modulus of the second Taylor coefficient is bounded in $F$, we define the order $\alpha$ of the linear-invariant family to be

$$
\alpha:=\frac{1}{2} \sup _{f \in F}\left|f^{\prime \prime}(0)\right| \text {. }
$$

An example of a linear-invariant family of order 2 is the class $S$ of normalized schlicht functions on the unit disk.

Pommerenke [9] (pp. 115-116) generalized the well-known Bieberbach distortion theorems [2] (see [12] p. 178) for $S$ to the concept of linear-invariant families and showed for a linear-invariant family $F$ of order $\alpha$ the relations

$$
\left\{\begin{array}{l}
|f(z)| \leqq \frac{1}{2 \alpha}\left(\left(\frac{1+|z|}{1-|z|}\right)^{\alpha}-1\right), \\
\left|f^{\prime}(z)\right| \leqq \frac{(1+|z|)^{\alpha-1}}{(1-|z|)^{\alpha+1}} .
\end{array}\right.
$$


We want to give further examples of linear-invariant families. Let $V_{k}$ be the class of functions of bounded boundary rotation $k \pi$ (see Lehto [7])

$$
V_{k}:=\left\{f \in N \mid \forall r \in\left[0,1\left[\left[\int_{0}^{2 \pi}\left|\operatorname{Re}\left(1+z \frac{f^{\prime \prime}}{f^{\prime \prime}}\right)\right| d \vartheta \leqq k \pi\right], \quad z=r e^{i \vartheta}\right\}\right.\right.
$$

for $k \in\left[2, \infty\left[\right.\right.$. Let further $C_{\beta}$ be the class of close-to-convex functions of order $\beta$ defined by Reade [11] and Pommerenke [10],

$$
C_{\beta}:=\left\{f \in N \mid \exists \varphi \text { schlicht with convex range }\left[\left|\arg \frac{f^{\prime}}{\varphi^{\prime}}\right| \leqq \beta \frac{\pi}{2}\right]\right\},
$$

for $\beta \in[0, \infty[$.

Properties of these classes are given in the book of Schober [12] (Chapter 2).

As special cases we have $V_{2}=C_{0}$, the well-known class of normalized convex functions, and $C_{1}$, the class of close-to-convex functions defined by Kaplan [5]. The classes $V_{k}$ and $C_{\beta}$ are increasing in $k$ and $\beta$, respectively, and until $k=4$ and $\beta=1$ they contain only schlicht functions.

Aharonov and Friedland [1] showed that the Taylor coefficients of functions in $C_{\beta}$ as well as in $V_{k}$ are dominated in modulus by the corresponding coefficients of the function $h_{\alpha}$ defined by

$$
h_{\alpha}(z):=\frac{1}{2 \alpha}\left(\left(\frac{1+z}{1-z}\right)^{\alpha}-1\right)
$$

with $\alpha:=k / 2$ resp. $\alpha:=\beta+1$. That means: For $f \in V_{2 \alpha}$ or $f \in C_{\alpha-1}$ we have

$$
\left|f^{(n)}(0)\right| \leqq h_{\alpha}^{(n)}(0) \text {. }
$$

In the proof of this inequality they used the inclusion

$$
V_{2 \alpha} \subset C_{\alpha-1} .
$$

As closed normal families all classes $V_{k}$ and $C_{\beta}$ are compact with respect to the topology of locally uniform convergence.

Now we prove the linear-invariance of these classes.

2. Lemma. For every $\beta \in\left[0, \infty\left[\right.\right.$ the family $C_{\beta}$ is linear-invariant of order $\beta+1$. For every $k \in\left[2, \infty\left[\right.\right.$ the family $V_{k}$ is linear-invariant of order $k / 2$.

Proof. Reade [11] and Pommerenke [10] showed the desired property for $C_{\beta}$ if $\beta \in[0,1]$. In this case the functions are all schlicht and so this property follows from a geometrical description of the classes.

We now take an arbitrary $\beta \in\left[0, \infty\left[\right.\right.$. Let $f \in C_{\beta}$ with convex $\varphi$ such that

$$
\left|\arg \frac{f^{\prime}}{\varphi^{\prime}}\right| \leqq \beta \frac{\pi}{2} \text {. }
$$


Our first step will be to show that $C_{\beta}$ has the rotation-invariance property, which means

whenever $|x|=1$ and

$$
f \in C_{\beta} \Rightarrow f_{x} \in C_{\beta}
$$

$$
f_{x}(z):=\frac{f(x z)}{x}
$$

The function $\varphi_{1}$ defined by $\varphi_{1}(z):=\varphi(x z) / x$ has convex range and obeys the inequality

$$
\left|\arg \frac{f_{x}^{\prime}}{\varphi_{1}^{\prime}}\right| \leqq \beta \frac{\pi}{2}
$$

So $C_{\beta}$ inherits this property from $C_{0}$. We show now that $C_{\beta}$ inherits the linear-invariance property, too. Therefore it is enough to show that for

$$
l(z)=\frac{z+r}{1+r z} \quad \text { with } \quad r \in[0,1[
$$

and for $f \in C_{\beta}$ also the function

$$
g:=\frac{f \circ l-f \circ l(0)}{(f \circ l)^{\prime}(0)}
$$

is in $C_{\beta}$. Now we have to find a convex $\varphi_{2}$ with

$$
\left|\arg \frac{g^{\prime}(z)}{\varphi_{2}^{\prime}(z)}\right| \leqq \beta \frac{\pi}{2} \text {. }
$$

We get

$$
\left|\arg \frac{g^{\prime}(z)}{\varphi_{2}^{\prime}(z)}\right|=\left|\arg \frac{f^{\prime}(l) l^{\prime}(z)}{f^{\prime}(r) \varphi_{2}^{\prime}(z)}\right|=\left|\arg \frac{f^{\prime}(l)}{\varphi_{2}^{\prime}(l)}+\arg \frac{\varphi_{2}^{\prime}(l) l^{\prime}(z)}{\varphi_{2}^{\prime}(z) f^{\prime}(r)}\right| .
$$

Since $f$ is in $C_{\beta}$, this expression will be less than or equal to $\beta \pi / 2$ if we take

$$
\varphi_{2}:=\frac{\varphi \circ l}{f^{\prime}(r)} \text {. }
$$

One sees from the geometric definition that the convexity of $\varphi$ implies the convexity of $\varphi_{2}$.

The order is given by the coefficient domination theorem (3).

In the case of the families $V_{k}$ the same argumentation gives the order. The linear-invariance property is a consequence of the geometrical interpretation of the definition. Because the ranges of $f$ and $g$ are similar, the limit boundary rotation of the two functions coincide,

$$
\lim _{r \rightarrow 1} \int_{0}^{2 \pi}\left|\operatorname{Re}\left(1+\frac{z f^{\prime \prime}}{f^{\prime}}\right)\right| d \vartheta=\lim _{r \rightarrow 1} \int_{0}^{2 \pi}\left|\operatorname{Re}\left(1+\frac{z g^{\prime \prime}}{g^{\prime}}\right)\right| d \vartheta
$$

since the integrals are monotone in $r$ (see [8], p. 12) and the suprema are equal. Lehto [7] (p. 12) already used the linear-invariance of $V_{k}$. 
Now we come to our main result.

3. Theorem. Let $\alpha \in[1, \infty[$, let $F$ be a linear-invariant family of order $\alpha$ and $n \geqq 2$. If for all $f \in F$ and all $m, 2 \leqq m \leqq n$,

$$
\left|f^{(m)}(0)\right| \leqq h_{\alpha}^{(m)}(0),
$$

then the corresponding distortion theorems

$$
\left|f^{(m)}\left(r e^{i \vartheta}\right)\right| \leqq h_{\alpha}^{(m)}(r)
$$

hold for all $r \in[0,1[$ and all $\vartheta \in \boldsymbol{R}$.

In particular we get for all linear-invariant families of order $\alpha$

$$
\left|f^{\prime \prime}\left(r e^{i \vartheta}\right)\right| \leqq 2(\alpha+r) \frac{(1+r)^{\alpha-2}}{(1-r)^{\alpha+2}}=h_{\alpha}^{\prime \prime}(r) .
$$

Proof. We generalize a result due to Landau [6] (see [12], p. 179).

We want to transform the information about $\left|f^{(m)}(0)\right|$ from the origin to an arbitrary point. Every linear-invariant family is of course rotation-invariant, and so we only need to consider a positive real point $r$.

Let be $f \in F$ and $l$ the Möbius-transform with

and let $g$ be the composition

$$
l(z)=\frac{z+r}{1+r z}
$$

If $g$ has the expansion

$$
g=f \circ l
$$

we get for $f$

$$
g(z)=\sum_{m=0}^{\infty} c_{m} z^{m}
$$

$$
f(z)=g \circ l^{-1}(z)=g\left(\frac{z-r}{1-r z}\right)=\sum_{m=0}^{\infty} c_{m}\left(\frac{z-r}{1-r z}\right)^{m} .
$$

Because of the generalized product rule

and the formula

$$
f=u v \Rightarrow f^{(n)}=\sum_{k=0}^{n}\left(\begin{array}{l}
n \\
k
\end{array}\right) u^{(k)} v^{(n-k)}
$$

we get

$$
\left.\left[(z-r)^{m}\right]^{(n)}\right|_{z=r}=n ! \delta_{n m}
$$

and further

$$
f^{(n)}(r)=\left.\sum_{m=0}^{n} c_{m}\left(\begin{array}{l}
n \\
m
\end{array}\right) m !\left[(1-r z)^{-m}\right]^{(n-m)}\right|_{z=r}
$$

$$
f^{(n)}(r)=n ! \sum_{m=1}^{n} c_{m} r^{n-m}\left(\begin{array}{c}
n-1 \\
m-1
\end{array}\right)\left(1-r^{2}\right)^{-n}
$$


Because of the linear-invariance property it follows from $f \in F$ that

$$
\frac{g-g(0)}{g^{\prime}(0)} \in F
$$

and so the given coefficient estimate shows

$$
\left|c_{m}\right| \leqq \frac{h_{\alpha}^{(m)}(0)}{m !}\left|g^{\prime}(0)\right| \text { for all } m \leqq n .
$$

If we take (5) with $n:=1$ we get

$$
\left|c_{1}\right|=\left|g^{\prime}(0)\right|=\left(1-r^{2}\right)\left|f^{\prime}(r)\right| .
$$

At that stage we utilize the linear-invariance property for the second time, using the distortion theorem (2) for the first derivative. Sc we get

$$
\left|c_{m}\right| \leqq \frac{h_{\alpha}^{(m)}(0)}{m !}\left(\frac{1+r}{1-r}\right)^{\alpha} \text { for all } m \leqq n
$$

and

$$
\left|f^{(n)}(r)\right| \leqq n ! \sum_{m=1}^{n} \frac{h_{\alpha}^{(m)}(0)}{m !}\left(\frac{1+r}{1-r}\right) r^{n-m}\left(\begin{array}{c}
n-1 \\
m-1
\end{array}\right)\left(1-r^{2}\right)^{-n}
$$

(as all terms here are positive). We shall show that the right-hand term equals $h_{\alpha}^{(n)}(r)$. With $f:=h_{\alpha}$ we get

$$
h_{\alpha} \circ l(z)=\frac{1}{2 \alpha}\left(\left(\frac{1+l(z)}{1-l(z)}\right)^{\alpha}-1\right)=\frac{1}{2 \alpha}\left(\left(\frac{1+r}{1-r}\right)^{\alpha}\left(\frac{1+z}{1-z}\right)^{\alpha}-1\right)
$$

and we write

$$
h_{\alpha} \circ l(z)=A h_{\alpha}(z)+B
$$

with

$$
\begin{gathered}
A=\left(\frac{1+r}{1-r}\right)^{\alpha}, \\
B=h_{\alpha}(r) .
\end{gathered}
$$

So we have

$$
h_{\alpha} \circ l^{(m)}(0)=\left(\frac{1+r}{1-r}\right)^{\alpha} h_{\alpha}^{(m)}(0),
$$

and the right-hand side of (6) gets the form

$$
n ! \sum_{m=1}^{n} \frac{h_{\alpha} \circ l^{(m)}(0)}{m !} r^{n-m}\left(\begin{array}{c}
n-1 \\
m-1
\end{array}\right)\left(1-r^{2}\right)^{-n} .
$$

Looking back to formula (5) we see that this is an expression for $h_{\alpha}^{(n)}(r)$. So we get our conclusion for the index $m:=n$. For $m<n$ the proof coincides with the given one and our result follows. 
In the special case $n:=2$ we get the distortion theorem because of the definition of the order. (Bieberbach was the first who proved this distortion theorem in the class $S$ [3].)

4. Corollary. Let $\alpha \in\left[1, \infty\left[\right.\right.$ and $n \in N_{0}$. Then the following equality holds:

$$
\max _{f \in C_{\alpha-1}} \max _{\vartheta \in R}\left|f^{(n)}\left(r e^{i \vartheta}\right)\right|=\max _{f \in V_{2 \alpha}} \max _{\vartheta \in R}\left|f^{(n)}\left(r e^{i \vartheta}\right)\right|=h_{\alpha}^{(n)}(r) .
$$

Proof. Because of the compactness of the classes the maximum exists. Formulae (2) for $n \in\{0,1\}$ and our theorem for $n \geqq 2$ show what maximum we can hope to get.

The well-known results

make the results sharp.

$$
h_{\alpha} \in V_{2 \alpha} \text { and } h_{\alpha} \in C_{\alpha-1}
$$

5. Remark. The theorem we proved shows that the linear-invariance property helps us to obtain successive distortion theorems for the $n$-th derivative in an arbitrary linear-invariant family from the corresponding coefficient estimates.

But if we have - as in the cases $C_{\beta}$ and $V_{k}$ - the coefficient estimates for all $n$, we can get the distortion theorems more elementarily and without using the linear-invariance property from the following Lemma.

The Lemma arises from a note of Doppel and Volkmann [4], who used it solving a similar problem for another class.

6. Lemma. Let in the unit disk

and

$$
f(z)=\sum_{n=0}^{\infty} a_{n} z^{n}
$$

with $b_{n} \in[0, \infty[$ for all $n$. If

$$
g(z)=\sum_{n=0}^{\infty} b_{n} z^{n}
$$

holds for all $n$, we get

$$
\left|a_{n}\right| \leqq b_{n}
$$

for all $z$ in the unit disk.

$$
\left|f^{n}(z)\right| \leqq g^{(n)}(|z|)
$$

Proof. The identity

$$
f^{(n)}(z)=n ! \sum_{k=n}^{\infty}\left(\begin{array}{l}
k \\
n
\end{array}\right) a_{k} z^{k-n}
$$

ane the corresponding one for $g$ imply

$$
\begin{gathered}
\left|f^{n}(z)\right|=n !\left|\sum_{k=n}^{\infty}\left(\begin{array}{l}
k \\
n
\end{array}\right) a_{k} z^{k-n}\right| \leqq n ! \sum_{k=n}^{\infty}\left|\left(\begin{array}{l}
k \\
n
\end{array}\right) a_{k} z^{k-n}\right|=n ! \sum_{k=n}^{\infty}\left(\begin{array}{l}
k \\
n
\end{array}\right)\left|a_{k}\right||z|^{k-n} \\
\leqq n ! \sum_{k=n}^{\infty}\left(\begin{array}{l}
k \\
n
\end{array}\right) b_{k}|z|^{k-n}=g^{(n)}(|z|) .
\end{gathered}
$$




\section{References}

[1] Aharonov, D., and S. Friedland: On an inequality connected with the coefficient conjecture for functions of bounded boundary rotation. - Ann. Acad. Sci. Fenn. Ser. A. I. Math. 524, 1973, 1-13.

[2] Bieberbach, L.: Über die Koeffizienten derjenigen Potenzreihen, welche eine schlichte Abbildung des Einheitskreises vermitteln. - Sitzungsberichte der Königl. Preußischen Akademie der Wissenschaften 1916, 940-955.

[3] Bieberbach, L.: Aufstellung und Beweis des Drehungssatzes für schlichte konforme Abbildungen. - Math. Z. 4, 1919, 295-305.

[4] Doppel, K., und L. Volkmann: Koeffizientenabschätzung für Funktionen, deren erste Ableitung beschränktes Argument hat. - Ann. Acad. Sci. Fenn. Ser. A. I. Math. 4, 1978/1979, 259-265.

[5] Kaplan, W.: Close-to-convex schlicht functions. - Michigan Math. J. 1, 1952, 169-185.

[6] Landau, E.: Einige Bemerkungen über schlichte Abbildung. - Jahresber. Deutsch. Math.Verein. 34, 1925/26, 239-243.

[7] Lehto, O.: On the distortion of conformal mappings with bounded boundary rotation. Ann. Acad. Sci. Fenn. Ser. A. I. Math.-Phys. 124, 1952, 1-14.

[8] PaAtero, V.: Über die konforme Abbildung von Gebieten deren Ränder von beschränkter Drehung sind. - Ann. Acad. Sci. Fenn. Ser. A. 33:9, 1931, 1-78.

[9] Pommerenke, Ch.: Linear-invariante Familien analytischer Funktionen I. - Math. Ann. $155,1964,108-154$.

[10] Pommerenke, Ch.: On close-to-convex analytic functions. - Trans. Amer. Math. Soc. 114, 1965, 176-186.

[11] REAde, M. O.: The coefficients of close-to-convex functions. - Duke Math. J. 23, 1956, 459-462.

[12] Schober, G.: Univalent functions - Selected topics. - Lecture Notes in Mathematics 478, Springer-Verlag, Berlin-Heidelberg-New York, 1975.

Freie Universität Berlin

Fachbereich Mathematik

Arnimallee 2-6

D 1000 Berlin 33

Bundesrepublik Deutschland

Received 21 April 1983 